\title{
Valoración de una situación didáctica para la enseñanza de variable aleatoria y distribución de probabilidad en la educación secundaria chilena
}

\author{
Assessment of a didactic situation for teaching the random variable \\ and probability distribution in Chilean secondary education \\ Avaliação de uma situação didática para o ensino da variável aleatória \\ e a distribuição de probabilidade na educação secundária chilena
}

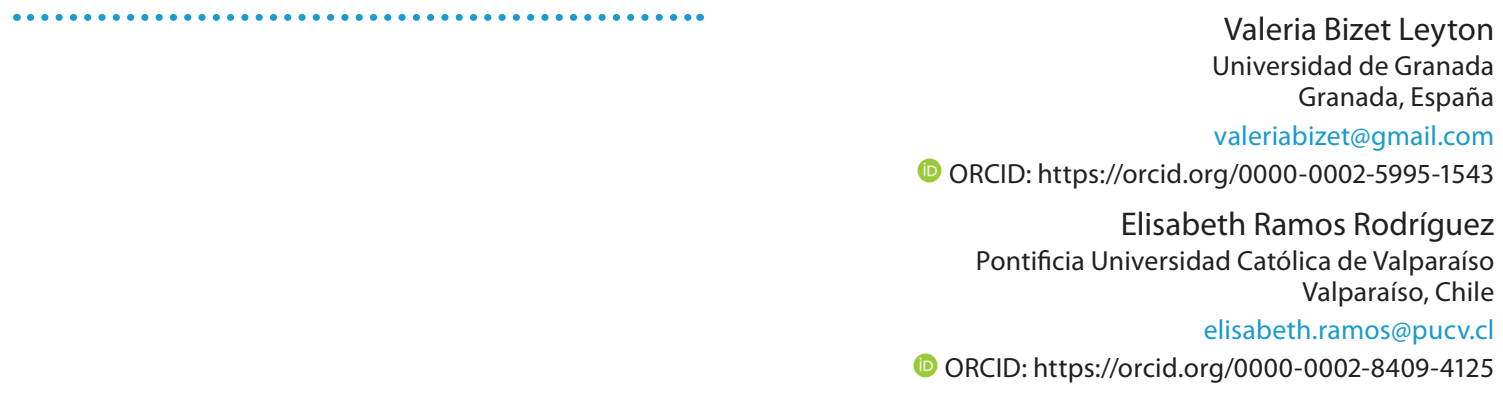

Recibido - Received - Recebido: 15 / 09 / 2021 Corregido - Revised - Revisado: 29 / 10 / 2021 Aceptado - Accepted - Aprovado: 12 / 11 / 2021

DOI: https://doi.org/10.22458/ie.v24i36.3897

URL: https://revistas.uned.ac.cr/index.php/innovaciones/article/view/3897

\begin{abstract}
Resumen: La variable aleatoria y su distribución de probabilidad son conceptos fundamentales de tratar en la educación estadística y probabilística escolar, debido a que se manifiestan en experiencias cotidianas. Aun así, su enseñanza se hace compleja debido, prioritariamente, a las dificultades asociadas a la comprensión de estas y el concepto de función. Por tanto, se propone como objetivo de estudio valorar un proceso de instrucción de la variable aleatoria y su distribución de probabilidad en la educación secundaria chilena, fundamentado en la Teoría de Situaciones Didácticas (TSD) y la Teoría de Registro de Representaciones Semiótica (TRRS). Bajo la perspectiva cualitativa y con un enfoque descriptivo-interpretativo, se construye un test que contiene una situación didáctica, la cual fue aplicada a 22 estudiantes chilenos de grado 10 (15 a 16 años). Los hallazgos evidenciaron que más de la mitad de los estudiantes (64\%), logró identificar y representar en lenguaje verbal, figural o tabular, la variable aleatoria, y un porcentaje similar (59\%), su distribución de probabilidad. Desde la TRRS, esto podría indicar que los estudiantes están en vías de construcción de ambos objetos. Desde la TSD, se observa el importante rol que cumplió el profesor en las fases de una situación didáctica. Estudiar, desde teorías didácticas, cómo llevar al aula objetos complejos de enseñar, puede ayudar a profesores en ejercicio y en formación a mirar su práctica con mayor fundamento, para hacerla más accesible y comprensible a sus estudiantes.
\end{abstract}

Palabras claves: Variable aleatoria; distribución de probabilidad; teoría de la educación; enseñanza de la estadística; enseñanza secundaria, educación.

Summary: The random variable and its probability distribution are fundamental concepts in statistical and probabilistic school education because they manifest in everyday experiences.

Teaching them becomes a complex task due primarily to the difficulties associated with understanding them and the concept of function. Therefore, we proposed as a study objective to assess the random variable's instruction process and its probability distribution in Chilean secondary education, based on the Theory of Didactic Situations (TSD) and the Theory of Registration of Semiotic Representations (TRRS). 
We designed a test containing a didactic situation applied to 10th-grade Chilean students (15 to 16 years old) with a qualitative perspective and a descriptive-interpretive approach.

The findings showed that more than half of the students (64\%) managed to identify and represent the random variable in verbal, figural, or tabular language, and a similar percentage (59\%), its probability distribution. Concerning the TRRS, this could indicate that students are in the process of building both objects. Subsequently from the TSD, we observed the vital role that the teacher played in the phases of the said didactic situation. Studying didactic theories, we discovered that bringing complex objects to the classroom can help actual teachers and those being trained to look at their practice with more understructure to make it more accessible and understandable to their students.

Keywords: random variable; probability distribution; education theory; teaching of statistics; secondary education; education.

Resumo: A variável aleatória e a sua distribuição de probabilidade são conceitos fundamentais na educação estatística e probabilística escolar, devido a que se manifestam nas experiências cotidianas. Mesmo assim, seu ensino se torna complexo devido, prioritariamente às dificuldades associadas à compreensão delas e o conceito de função. Portanto, o que se propõe como objetivo de estudo é avaliar um processo de instrução da variável aleatória e sua distribuição de probabilidade na educação secundária chilena, fundamentado na Teoria das Situações Didáticas (TSD), e a Teoria de Registro de Representações Semióticas (TRRS). Sob uma perspectiva qualitativa e com uma abordagem descritiva-interpretativa, foi construído um teste que contém uma situação didática, e o qual foi aplicado a 22 estudantes chilenos da 10a série (15 a 16 anos). Os achados evidenciaram que mais da metade dos estudantes (64\%) conseguiu identificar e representar em linguagem verbal, gráfico ou tabular, a variável aleatória, e uma porcentagem similar (59\%) sua distribuição de probabilidade. Desde a TRRS, isto poderia indicar que os estudantes estão no processo de construção de ambos os objetos. Desde a TSD, observa-se o papel fundamental que teve o professor nas fases da situação didática. Estudar, desde as teorias didáticas, como levar para a sala de aula objetos complexos de se ensinar pode ajudar os professores tanto os que exercem quanto aqueles que estão em formação a terem um olhar mais fundamentado sobre a sua prática para que ela seja mais accessível e compreensível para os estudantes.

Palavras-chave: variável aleatória, distribuição da probabilidade, teoria da educação, ensino da estatística, ensino médio, educação.

\section{INTRODUCCIÓN}

Diversos autores señalan la problemática relativa a las dificultades que tienen los estudiantes de secundaria, para comprender la variable aleatoria y la distribución de probabilidad como funciones (Landín y Salinas, 2016; Salazar, 2014), teniendo en cuenta que la variable aleatoria y su distribución de probabilidad son conceptos fundamentales de tratar en la educación estadística y probabilística escolar, debido a que se manifiestan en experiencias cotidianas (Batanero, 2004; Heitele, 1975).

Esta relevancia queda de manifiesto en diversas directrices curriculares internacionales dirigidas a los grados 9 a 12 (14 a 18 años), como los Principios y Estándares para la Matemática Escolar de Estados Unidos (National Council of Teachers of Mathematics, 2000) y el currículo español (Ministerio de Educación Cultura y Deporte, 2015), que incluyen el estudio de la variable aleatoria, y sus funciones de probabilidad y distribución. Chile no queda ajeno a este escenario, dado que sus Bases Curriculares vigentes (Ministerio de Educación de Chile, 2015) en grado 10 (15 a 16 años) introduce los tópicos en cuestión.

Sin embargo, parece ser que las investigaciones sobre el tratamiento de la variable aleatoria y su distribución de probabilidad en educación escolar son escasas. Salazar (2014) indagó, a partir de un cuestionario, cómo estudiantes chilenos de los grados 9 a 12 (14 a 18 años) construían la variable aleatoria. Los resultados muestran falta del reconocimiento de la variable aleatoria como una función y problemas en la identificación de la relación entre los elementos del espacio muestral (su dominio) y números reales (su recorrido). También Flores, García y Sánchez (2014) analizan el razonamiento probabilístico sobre aquellos conceptos relacionados con la variable aleatoria, para lo cual aplicaron una actividad con apoyo de tecnología a 69 estudiantes mexicanos de grado 8 (13 a 14 años), considerando un pre y post test. 
En la segunda evaluación los resultados evidencian que solo el $60 \%$ propone una distribución de probabilidad cuyos valores se aproximan a las probabilidades teóricas y suman 1 o $100 \%$.

Por su parte, Landín y Salinas (2016) investigaron, mediante dos cuestionarios, el razonamiento en torno al espacio muestral y probabilidad de sucesos compuestos que llevan a cabo 28 estudiantes mexicanos de grado 12 (17 a 18 años). Entre los resultados del segundo cuestionario observaron que solo un $21 \%$ utiliza como estrategia de resolución el definir una variable aleatoria, aunque calcularon incorrectamente las probabilidades de sus valores; además, las representaciones más empleadas fueron tablas, listas y diagramas. Mientras Doukhan y Gueudet (2019) valoraron, a través de un cuestionario, la comprensión de los tópicos relacionados a la variable aleatoria, en estudiantes franceses de grado 10 (15 a 16 años). Entre sus resultados obtuvieron que, el $40 \%$ respondió erróneamente al definir variable aleatoria y dar un ejemplo, pues la confunden con su distribución, aunque el $85 \%$ determinó una función de probabilidad, algunos de ellos utilizaron la estrategia de representar la situación en un diagrama (sagital o de árbol).

En esta perspectiva, este trabajo aborda el problema relativo a las dificultades en secundaria para comprender la variable aleatoria y la distribución de probabilidad como funciones, fundamentado en dos teorías de la educación matemática: la Teoría de Situaciones Didácticas (TSD) y la Teoría de Registro de Representaciones Semiótica (TRRS).

Por un lado, la TSD (Brousseau, 2007) analiza el sistema didáctico integrado por tres elementos: el profesor, el estudiante y el saber, además de sus interacciones centradas en los aspectos cognitivos y epistemológicos de la construcción del conocimiento matemático. En este contexto, una situación es comprendida como "un modelo de interacción entre un sujeto y un medio determinado" (Brousseau, 2007, p. 17), en tanto que, para la realidad de la presente investigación, educación escolar, corresponde al entorno del estudiante, empleado en el proceso de enseñanza-aprendizaje, el cual ha sido creado y manipulado por la persona profesora.

Dentro de la TSD existe un elemento fundamental denominado situación a-didáctica, entendida como una situación donde sin intervención del profesor, el estudiante aprende por interacción con el medio, por medio de la aplicación de sus conocimientos antiguos, surge en él la necesidad de aprender una nueva temática. Aquella está constituida por las siguientes tres fases que conducen gradualmente al estudiante a especificar el conocimiento utilizado para resolver un problema (Brousseau, 1998), a saber:

1. Situación de acción: primera interacción del estudiante con el medio; él, mediante la puesta en acción de sus conocimientos previos y elaboración de estrategias, intenta responder al problema propuesto.

2. Situación de formulación: en esta instancia, al menos dos estudiantes se vinculan con el medio, cada uno comunica a los integrantes de su equipo la estrategia de resolución y es discutida para crear una común.

3. Situación de validación: el estudiante aprende cómo convencer a sus pares, es consciente de las razones que propone para convencer a otros, o las que acepta para cambiar su punto de vista son aceptadas progresivamente, puestas a prueba, debatidas y convenidas.

En la situación a-didáctica, el profesor se restringe a alentar al estudiante a resolver un problema, hacer que identifique las acciones que son posibles de llevar a cabo sobre el medio y decidir si lo ha lograrlo. El producto de esta situación es un conocimiento, que en el contexto escolar es interpretado como una estrategia de resolución. Debido a que el estudiante no siempre logra resolver un problema, es el profesor quien debe identificar aquellos que están a su alcance. En este caso, surge la situación didáctica, situación elegida por el profesor, que lo involucra a él en un juego con el sistema de interacciones del estudiantado con su medio (Brousseau, 2007), por lo que una situación a-didáctica es parte de una situación didáctica y su interacción se aprecia en la Figura 1. 
Figura 1. Relación entre situación didáctica y a-didáctica

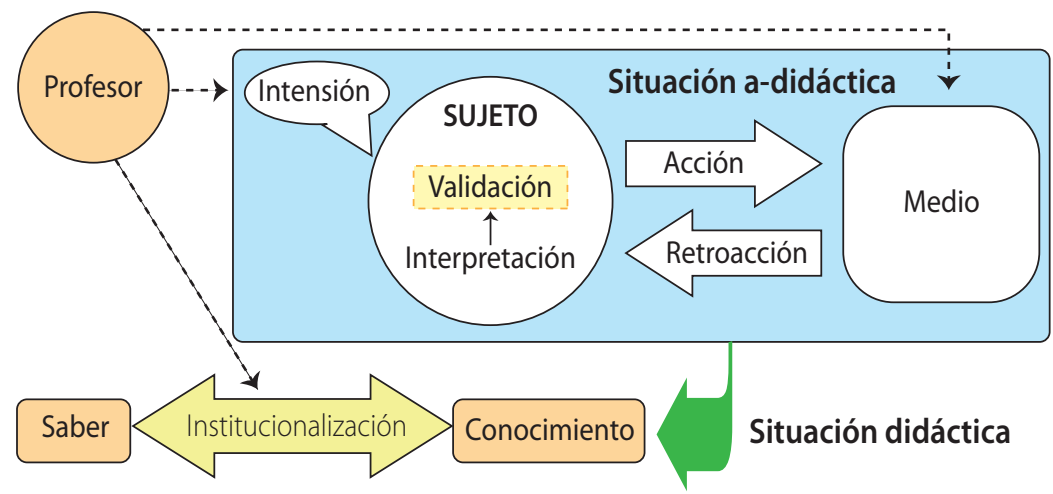

Fuente: Acosta, Monroy y Rueda (2010, p. 177).

Terminada la situación a-didáctica, es desarrollada una cuarta fase, la situación de institucionalización, momento en que el profesor explicita los vínculos entre el conocimiento construido previamente por el estudiante y el saber qué pretende enseñar (Brousseau, 1998). De esta manera, en el presente estudio se utilizaron las cuatro fases que componen una situación didáctica, para el diseño de un proceso de instrucción centrado en la resolución de un problema.

Otro referente teórico del estudio es la TRRS (Duval, 2006), teoría que analiza el ámbito cognitivo del desarrollo del conocimiento matemático, donde uno de sus fundamentos es la diversidad de registros de representación de un objeto matemático, tales como: lenguaje verbal, lenguaje tabular, lenguaje figural, lenguaje gráfico, lenguaje simbólico, entre otros. En esta perspectiva, la construcción de un concepto en matemática está determinada por la capacidad del estudiante de transitar entre al menos dos registros, donde aquella actividad vinculada a producir una representación es denominada semiosis y la relacionada a la asimilación conceptual en torno a un objeto matemático, es llamada noesis.

Particularmente la TRRS en esta investigación fue empleada en la identificación de las diversas estrategias de resolución del problema diseñado. Debido a que esta aborda los conceptos de variable aleatoria y su distribución de probabilidad, los cuales son funciones, entonces se basa cada estrategia en algún registro de representación de este objeto matemático (Prada-Nuñez, Hernández-Suárez y Jaimes, 2017): lenguaje verbal, tabular y figural.

De esta forma, para abordar el problema relativo a las dificultades que tienen los estudiantes de secundaria para comprender la variable aleatoria y la distribución de probabilidad como funciones, se plantea como objetivo del presente estudio, valorar un proceso de instrucción de la variable aleatoria y su distribución de probabilidad en educación secundaria.

\section{MATERIALES Y MÉTODOS}

Esta investigación fue desarrollada desde una perspectiva cualitativa y es de tipo descriptivo-interpretativo (Hernández, Fernández y Baptista, 2006). Los sujetos informantes fueron 22 estudiantes chilenos de grado 10 (15 a 16 años), pertenecientes a una escuela subvencionada por el Ministerio de Educación de un estrato socioeconómico medio. Este grupo fue elegido de manera intencionada y bajo criterio de disponibilidad y accesibilidad, donde su profesor de matemática fue quien llevó a cabo el proceso de instrucción en cuestión; además, es quien diseñó y refinó la propuesta de aula que se implementó. 
El instrumento utilizado para la recogida de datos, fue un test que contenía un problema (situación didáctica) diseñado por un profesor de matemática que tiene conocimiento de la TSD y la TRRS. El problema, integrado por dos interrogantes (ver Figura 2, izquierda), fue sometido a un proceso de validación de tres expertos en Educación Matemática, doctores en esta área, quienes, en conjunto en una reunión, decidieron que solo la pregunta uno no resolvía este y podía plantearse la dos por sí sola, pero modificada (ver Figura 2, derecha).

Figura 2. Instrumento inicial de recogida de datos

\section{Problema}

A raíz de los festejos del día del alumno, el centro de padres del Colegio Sol Naciente desea conocer el número de estudiantes que tiene cada uno de los 200 apoderados del colegio, solicita la información a la secretaria del establecimiento. Los resultados para tal efecto son:

\begin{tabular}{|l|c|c|c|c|c|c|c|c|c|}
\hline Número de apoderados & 20 & 35 & 47 & 33 & 22 & 17 & 12 & 9 & 5 \\
\hline Número de estudiantes & 1 & 2 & 3 & 4 & 5 & 6 & 7 & 8 & 9 \\
\hline
\end{tabular}

La intención es efectuar una rifa que beneficie a los alumnos, se asignará a cada apoderado un boleto de rifa. En la celebración del día del alumno, se realizará el sorteo y se premiará a los estudiantes de un apoderado con entradas para el cine, pero estas se tienen que comprar con anticipación, pues hasta mañana están en oferta. Así que se recomienda al centro general de padres del colegio, que decida cuántas tiene que comprar, con la finalidad de abaratar costos.

Interrogantes propuestas a expertos

1. ¿Cuáles son los posibles casos que podrían ocurrir? ¿Cuál de estas es más posible que ocurra? ¿Por qué?

2. Dados los conjuntos $A, B$ y $C$ definidos por

A: el conjunto de 200 apoderados del colegio

B: el conjunto de cantidad de estudiantes

C: el conjunto de posible ocurrencia de cada situación

Defina y represente la relación entre los conjuntos.

\section{Interrogante sugerida por los expertos}

Dados los conjuntos A, B y C definidos por

A: el conjunto de 200 apoderados del colegio

B: el conjunto de cantidad de estudiantes

C: el conjunto de posible ocurrencia de cada situación Defina y represente la relación entre $A$ y $B$ y entre $B$ y $C$.

Fuente: Elaboración propia.

Posteriormente se llevó a cabo una prueba piloto del instrumento en un grupo reducido de nueve estudiantes chilenos de grado 10 (15 a 16 años), quienes manifestaron como principal dificultad la de representar el conjunto A, debido a la cantidad de elemento que poseía.

De esta manera fue reformulado el instrumento (Figura 3), obteniendo la versión final del problema, cuyo propósito es tratar los conceptos de variable aleatoria y su distribución de probabilidad a nivel escolar. Para ello, es necesario que el estudiante primero reconozca y represente la relación entre los conjuntos A y B, además de la correspondencia entre los conjuntos B y C. Luego, identifique que las relaciones son funciones $y$, finalmente, mediante la intervención del profesor, establezca que en el contexto de probabilidad aquellas son denominadas variable aleatoria y distribución de probabilidad respectivamente. Así es introducido el estudio de nuevos conceptos probabilísticos, la variable aleatoria y su vínculo directo con la distribución de probabilidad. La propuesta de aula se diseñó considerando las habilidades propuestas por el Ministerio de Educación de Chile (2016), para los tópicos probabilísticos en cuestión, estas son: organizar, analizar y hacer inferencias acerca de información representada en tabla, elaborar representaciones de acuerdo con las necesidades del problema, y transitar entre los distintos registros de representación de funciones. 
Figura 3. Instrumento final de recogida de datos

\section{Problema}

A raíz de los festejos del día del alumno, el profesor de taller de cine del Colegio Sol Naciente desea conocer el número de estudiantes inscritos que tiene cada uno de los 30 apoderados del taller, por lo cual solicita la información a la secretaria del establecimiento. Los resultados para tal efecto son:

\begin{tabular}{|l|c|c|c|c|}
\hline Número de apoderados & 8 & 13 & 7 & 2 \\
\hline Número de estudiantes & 1 & 2 & 3 & 4 \\
\hline
\end{tabular}

La intención es efectuar una rifa que beneficie a los alumnos, se asignará a cada apoderado un boleto de rifa.

En la celebración del día del alumno, se realizará el sorteo y se premiará a los estudiantes de un apoderado con entradas para el cine, pero estas se tienen que comprar con anticipación, pues hasta mañana están en oferta. Por lo tanto, el profesor debe decidir cuántas tiene que comprar, con la finalidad de abaratar costos.
Dados los conjuntos A, B y C definidos por
A: el conjunto de 30 apoderados del taller.
B: el conjunto de cantidad de estudiantes.
C: el conjunto de posible ocurrencia de cada situación
Defina y represente la relación entre $A$ y $B$ y entre $B$ y $C$.

Fuente: Bizet (2017, p. 29).

Para el análisis exhaustivo de las respuestas proporcionadas por los participantes, fue empleada la técnica de análisis de contenido (Flick, 2004). Las categorías de análisis $\left(C_{n}\right)$ se establecieron mediante un análisis a priori del problema desde la TSD. Específicamente, fueron inferidas a partir de los conceptos matemáticos y distintas estrategias puestas en juego en su resolución, según las fases de la TSD en el desarrollo del proceso de instrucción, es decir, la situación de acción y la situación de formulación (momentos en que el estudiante posee el protagonismo).

En la primera fase del proceso de instrucción, situación acción, las categorías fueron definidas a partir del reconocimiento de los conceptos que contribuyen a resolver el problema, así se establecieron cuatro: $C_{1}$, vinculada a los conceptos de experimento aleatorio y espacio muestral; $C_{2}$, relacionada con la partición de un conjunto y sucesos aleatorios compuestos; $C_{3}$, asociada a la asignación de probabilidad desde su enfoque clásico/teórico (Regla de Laplace); $C_{4}$, sobre función, su dominio y recorrido. La Tabla 1 describe cada una de estas.

TABLA 1

Categorías de análisis de la situación de acción

\begin{tabular}{|c|c|}
\hline Categorías & Descripción \\
\hline $\begin{array}{l}\mathrm{C}_{1} \text { Identificación del espacio muestral asociado al } \\
\text { experimento }\end{array}$ & $\begin{array}{l}\text { El estudiante determina todos los elementos del conjunto A como una } \\
\text { colección de elementos, representado, por ejemplo } \\
A=\{\text { apoderado } 1, \ldots \text {, apoderado } 30\}\end{array}$ \\
\hline $\begin{array}{l}\mathrm{C}_{2} \text { Clasificación de los elementos del espacio } \\
\text { muestral, según la característica del problema }\end{array}$ & $\begin{array}{l}\text { El estudiante agrupa los elementos del espacio muestral, según la } \\
\text { cantidad de estudiantes que posee un apoderado }\end{array}$ \\
\hline $\begin{array}{l}\mathrm{C}_{3} \text { Identificación que los elementos del conjunto } \mathrm{C} \\
\text { son probabilidades }\end{array}$ & $\begin{array}{l}\text { El estudiante identifica los cuatro elementos del conjunto C y expresa } \\
\text { la probabilidad de ocurrencia de cada suceso }\end{array}$ \\
\hline $\begin{array}{l}\mathrm{C}_{4} \text { Reconocimiento que la relación entre los con- } \\
\text { juntos } \mathrm{A} \text { y } \mathrm{B} \text {, está representada en la tabla dada }\end{array}$ & $\begin{array}{l}\text { El estudiante afirma que el conjunto A está representado en la primera } \\
\text { fila de la tabla y el conjunto } B \text { en la segunda fila }\end{array}$ \\
\hline
\end{tabular}


Para la segunda fase del proceso de instrucción, situación de formulación, las categorías han sido establecidas desde la identificación de diversas estrategias de resolución del problema. De esta manera, fueron propuestas tres estrategias que dieron origen a seis categorías de análisis: estrategia 1, representar en lenguaje verbal la relación entre los conjuntos $A$ y $B$, y $B$ y $C$, donde emergieron $C_{5}$ y $C_{8}$, respectivamente; estrategia 2, representar en lenguaje tabular la relación entre $A$ y $B$, y $B$ y $C$, que originaron a $C_{7}$ y $\mathrm{C}_{10}$, correspondientemente; estrategia 3, representar en lenguaje figural (diagrama sagital o esquema) la relación entre $A$ y $B$, y $B$ y $C$, donde surgieron $C_{6}$ y $C_{9}$, respectivamente. La descripción de cada categoría es expuesta en la Tabla 2.

TABLA 2

Categorías de análisis de la situación de formulación

\section{Categorías}

$\mathrm{C}_{5}$ Identificación y registro en lenguaje verbal de la relación entre los conjuntos $A$ y $B$

$\mathrm{C}_{6}$ Representación en lenguaje figural de la relación entre los conjuntos $\mathrm{A}$ y $\mathrm{B}$

$\mathrm{C}_{7}$ Representación en lenguaje tabular de la relación entre los conjuntos $\mathrm{A}$ y $\mathrm{B}$

$C_{8}$ Identificación y registro en lenguaje verbal de la relación entre los conjuntos $\mathrm{B}$ y $\mathrm{C}$

$\mathrm{C}_{9}$ Representación en lenguaje figural de la relación entre en los conjuntos $\mathrm{B}$ y $\mathrm{C}$

$\mathrm{C}_{10}$ Representación en lenguaje tabular de la relación entre los conjuntos $\mathrm{B}$ y $\mathrm{C}$

\section{Descripción}

El estudiante expresa en lenguaje cotidiano la característica o cualidad que permite vincular a cada suceso (elemental) con un valor numérico

El estudiante utiliza un diagrama sagital o esquema en el que representar los conjuntos $A$ y $B$, y relaciona cada elemento del conjunto $A$ (suceso elemental) con un único elemento de $B$ (valor de la variable $\{1,2,3,4\}$ )

El estudiante realiza una tabla de valores donde la primera columna corresponde al número de apoderados y la segunda columna al número de estudiantes

El estudiante expresa en lenguaje cotidiano la co-rrespondencia que permite asignar a cada valor de la variable aleatoria un valor de probabilidad

El estudiante utiliza un diagrama sagital o esquema en el que representar los conjuntos $B$ y $C$, y relaciona cada elemento del conjunto $B$ (valor de la variable aleatoria $\{1,2,3,4\})$ con un único elemento de $C$ (valor de probabilidad)

El estudiante realiza una tabla de valores donde la primera columna corresponde al número de estudiantes y la segunda columna a la probabilidad asociada

Fuente: Elaboración propia.

Cabe destacar que la situación didáctica contempla dos fases más: la de validación y la de institucionalización. Dado el papel de las fases anteriores, en estas se ocupan las categorías ya señaladas.

El test fue aplicado por un profesor de matemática en una clase de 90 minutos de duración. Los 22 estudiantes se organizaron en cinco grupos: $G_{1}\left(5\right.$ estudiantes), $G_{2}$ (5 estudiantes), $G_{3}$ (4 estudiantes), $G_{4}$ ( 3 estudiantes) y $\mathrm{G}_{5}$ (5 estudiantes), y la respuesta de cada ha sido clasificada de acuerdo con las 10 categorías de análisis expuestas en las Tablas 1 y 2.

\section{DISCUSIÓN DE RESULTADOS}

El estudio desarrollado permitió valorar un proceso de instrucción, en torno a la variable aleatoria y su distribución de probabilidad en estudiantes de secundaria, fundamentado en la Teoría de Situaciones Didácticas (TSD) y la Teoría de Registro de Representaciones Semiótica (TRRS). Este proceso parece ser una situación didáctica, dado que permitió a los diferentes actores de él, avanzar en el aprendizaje y la enseñanza de la variable aleatoria y función de probabilidad, en el sentido que permitió al profesor abandonar su rol de comunicador del saber y a los estudiantes, construir su propio conocimiento como una experiencia grupal y colaborativa, con la cual el docente pudo conectar el saber institucional. Los resultados se presentan en relación con cada una de las etapas de la situación didáctica que se puso en juego con los estudiantes. 


\section{Situación de acción}

Al comienzo de esta fase, cuatro grupos $G_{1}, G_{2}, G_{3}$ y $G_{5}$ ) reconocieron como espacio muestral un conjunto integrado por la cardinalidad de los cuatro sucesos aleatorios compuestos que lo conforman (cantidad de apoderados, según número de estudiantes matriculado), tal como se muestra en la Figura 4. Así fue evidenciada la identificación y la partición incorrecta del espacio muestral, aquella dificultad también ha sido manifestada en la investigación de Batanero (2001).

Figura 4. Reconocimiento del espacio muestral de $\mathrm{G}_{5}$ previo a plantear la devolución al grupo curso

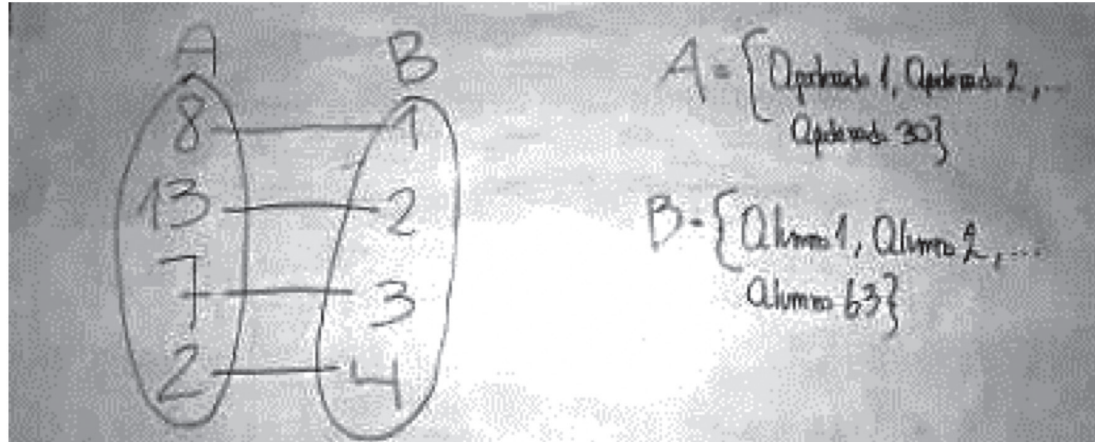

Fuente: Elaboración propia.

Para orientar el trabajo, el profesor hizo una pregunta de devolución al grupo de clase, la cual estuvo prevista en el diseño del proceso de instrucción: consideren este grupo de clase de grado 10 ¿Cómo representaría el conjunto de los estudiantes que utilizan lentes? ¿Cuáles son los elementos? ¿Quién puede representarlo en el pizarrón? Posteriormente, los grupos continuaron trabajando, logrando categorizar las producciones de cuatro grupos, según se muestra en la Tabla 3.

TABLA 3

Categorización de las respuestas de los grupos en la situación acción

\begin{tabular}{lcc}
\multicolumn{1}{c}{ Categorías } & Grupo & $\begin{array}{c}\text { Número(porcentaje) } \\
\text { de estudiantes }\end{array}$ \\
$\begin{array}{l}\mathrm{C}_{1} \text { Identificación del espacio muestral asociado al experimento } \\
\mathrm{C}_{2} \text { Clasificación de los elementos del espacio muestral según la caracte- } \\
\text { rística del problema }\end{array}$ & $\mathrm{G}_{1}-\mathrm{G}_{2}-\mathrm{G}_{3}-\mathrm{G}_{4}$ & $17(77 \%)$ \\
$\mathrm{C}_{3}$ Identificación que los elementos del conjunto $\mathrm{C}$ son probabilidades & $\mathrm{G}_{1}-\mathrm{G}_{2}-\mathrm{G}_{3}-\mathrm{G}_{4}$ & $17(77 \%)$ \\
$\begin{array}{l}\mathrm{C}_{4} \text { Reconocimiento que la relación entre los conjuntos } \mathrm{A} \text { y B está repre- } \\
\text { sentada en la tabla dada }\end{array}$ & $\mathrm{G}_{1}-\mathrm{G}_{2}-\mathrm{G}_{4}$ & $13(59 \%)$ \\
No realiza correctamente las acciones de $\mathrm{C}_{1} \mathrm{C}_{2} \mathrm{y} / \mathrm{o} \mathrm{C}_{3}$ & $\mathrm{G}_{2}$ & $5(23 \%)$ \\
\hline
\end{tabular}

Fuente: Elaboración propia.

En esta fase de la situación a-didáctica, se puede constatar el papel que jugó el docente al entregar una devolución acertada a los grupos para que estos pudiesen avanzar en esta fase. Las respuestas de cuatro grupos $\left(\mathrm{G}_{1}-\mathrm{G}_{2}-\mathrm{G}_{3}-\mathrm{G}_{4}\right)$ han sido clasificadas en la categoría $C_{1}$; in embargo, dos de estos $\left(\mathrm{G}_{1}\right.$ y $\left.\mathrm{G}_{4}\right)$ reconocieron el espacio muestral vinculado al experimento como una colección de números, constatado en la Figura 5. Por su parte, el grupo $\mathrm{G}_{5}$ entregó una respuesta incorrecta a lo solicitado, no pudiendo identificar el espacio muestral vinculado al experimento ni representar de alguna forma las relaciones solicitadas, lo que puede llevar a una reflexión en torno al papel que tiene el uso y manejo de diferentes registros (alentado por el docente en la pregunta de devolución), para el aprendizaje de la matemática, en especial para este tema que involucra el uso de funciones, como lo afirman Prada-Núñez et al. (2017). 
Figura 5. Identificación del espacio muestral en los grupos después de plantear devolución al grupo curso

$\mathrm{G}_{1}$

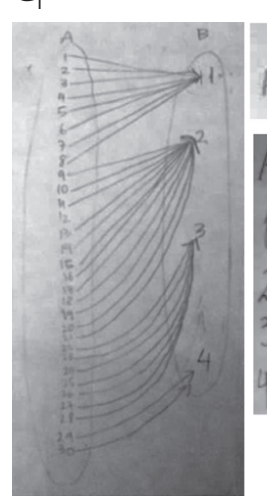

$\mathrm{G}_{2}$
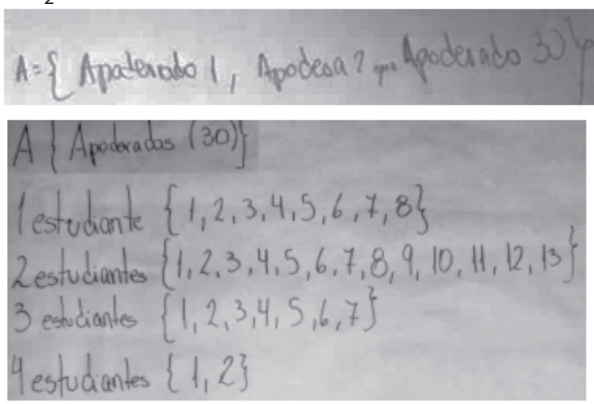

$\mathrm{G}_{3}$

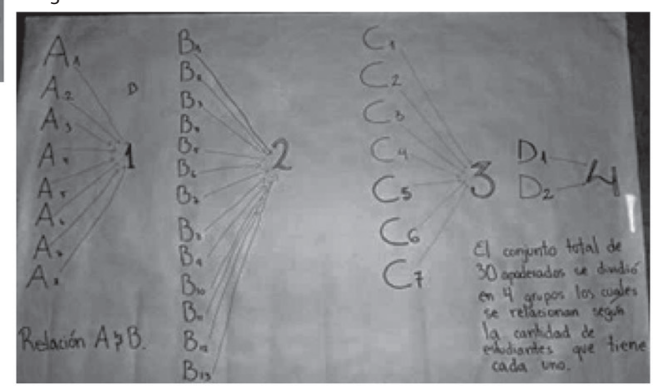

Fuente: Elaboración propia.

También tres grupos $\left(G_{1}, G_{2}, G_{4}\right)$ fueron clasificados en la categoría $C_{3}$, dado que identificaron que el conjunto $C$ está constituido por probabilidades de sucesos compuestos y establecieron sus cuatro elementos. Dos de ellos $\left(G_{2}\right.$ y $\left.G_{4}\right)$ representaron las probabilidades por medio de porcentajes y el otro $\left(G_{1}\right)$, como cociente entre el número de casos favorables del suceso y número de casos posibles, como se evidencia en la Figura 6. Aunque uno de los grupos no pudo avanzar satisfactoriamente en esta etapa, los resultados obtenidos sobre determinar la probabilidad de los valores de una variable aleatoria, son más alentadores (59\%) que los señalados por Landín y Salinas (2016). Un factor que pudo ser clave en ello, fue el conocimiento del profesor sobre el uso de representaciones para construir los conceptos (TRRS), y el manejo de buenas preguntas de devolución que hizo (TSD).

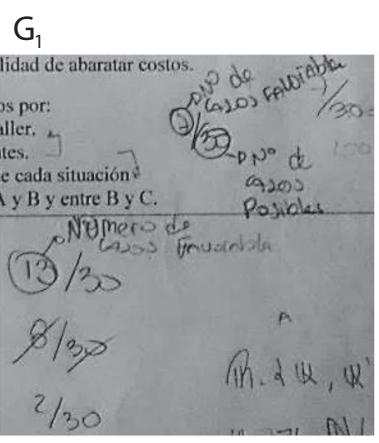

Figura 6. Identificación del conjunto $C$ en los grupos
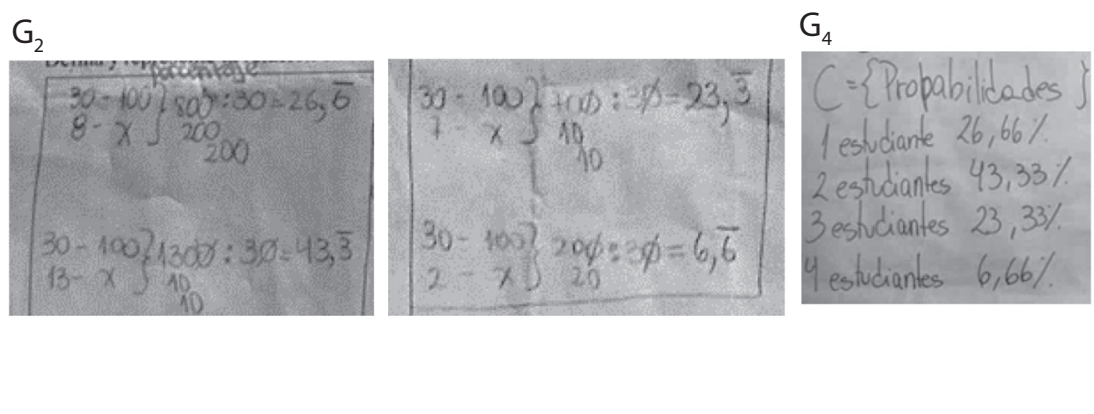

Fuente: Elaboración propia.

\section{Situación formulación}

En esta fase, se observaron en las producciones de los grupos las tres estrategias de resolución prevista en el análisis a priori del problema, por tanto, las respuestas han sido clasificadas como se muestra en la Tabla 4. 
TABLA 4

Categorización de las respuestas de los estudiantes en la situación formulación

\begin{tabular}{|c|c|c|}
\hline Categoría & Grupo & $\begin{array}{l}\text { Número (porcentaje) } \\
\text { de estudiantes }\end{array}$ \\
\hline $\begin{array}{l}C_{5} \text { Identificación y registro en lenguaje verbal de la relación entre los } \\
\text { conjuntos A y B }\end{array}$ & $\mathrm{G}_{1}-\mathrm{G}_{3}$ & $9(41 \%)$ \\
\hline $\begin{array}{l}\mathrm{C}_{6} \text { Representación en lenguaje figural de la relación entre los conjun- } \\
\text { tos } \mathrm{A} \text { y B }\end{array}$ & $\mathrm{G}_{1}-\mathrm{G}_{3}$ & $9(41 \%)$ \\
\hline $\begin{array}{l}C_{7} \text { Representación en lenguaje tabular de la relación entre los conjun- } \\
\text { tos } A \text { y B }\end{array}$ & $\mathrm{G}_{2}$ & $5(23 \%)$ \\
\hline $\begin{array}{l}C_{8} \text { Identificación y registro en lenguaje verbal de la relación entre los } \\
\text { conjuntos B y C }\end{array}$ & $\mathrm{G}_{1}-\mathrm{G}_{4}$ & $8(36 \%)$ \\
\hline $\begin{array}{l}C_{9} \text { Representación en lenguaje figural de la relación entre en los } \\
\text { conjuntos B y C }\end{array}$ & $\mathrm{G}_{1}$ & $5(23 \%)$ \\
\hline $\begin{array}{l}\mathrm{C}_{10} \text { Representación en lenguaje tabular de la relación entre los } \\
\text { conjuntos B y C }\end{array}$ & $\mathrm{G}_{2}$ & $5(23 \%)$ \\
\hline No representa la relación entre los conjuntos $A$ y $B, y / o$ B y $C$ & $\mathrm{G}_{5}$ & $5(23 \%)$ \\
\hline
\end{tabular}

Fuente: Elaboración propia.

Según es expuesto en la Tabla 4, tres grupos (64\%), $G_{1}, G_{2}, G_{3}$, identificaron en el problema la variable aleatoria, dos de estos $G_{1}$ y $G_{3}$ ) representaron la relación entre los conjuntos $A$ y $B$ en lenguaje verbal $\left(C_{5}\right)$ y lenguaje figural $\left(C_{6}\right)$, mediante un esquema o diagrama sagital. Además, solo el grupo $G_{2}$ representó aquella relación por medio de una tabla $\left(C_{7}\right)$, como se evidencia en la Figura 7. En tanto que $G_{5}$, dado que no pudo avanzar en la fase de acción, tampoco logró reconocer tal variable aleatoria. De esta manera, es posible constatar mejores resultados que los obtenidos por Salazar (2014), pues, en general, los estudiantes lograron representar la relación entre el espacio muestral (dominio de la variable aleatoria) y números reales (su recorrido).

Figura 7. Representación de la relación entre los conjuntos A y B en los grupos
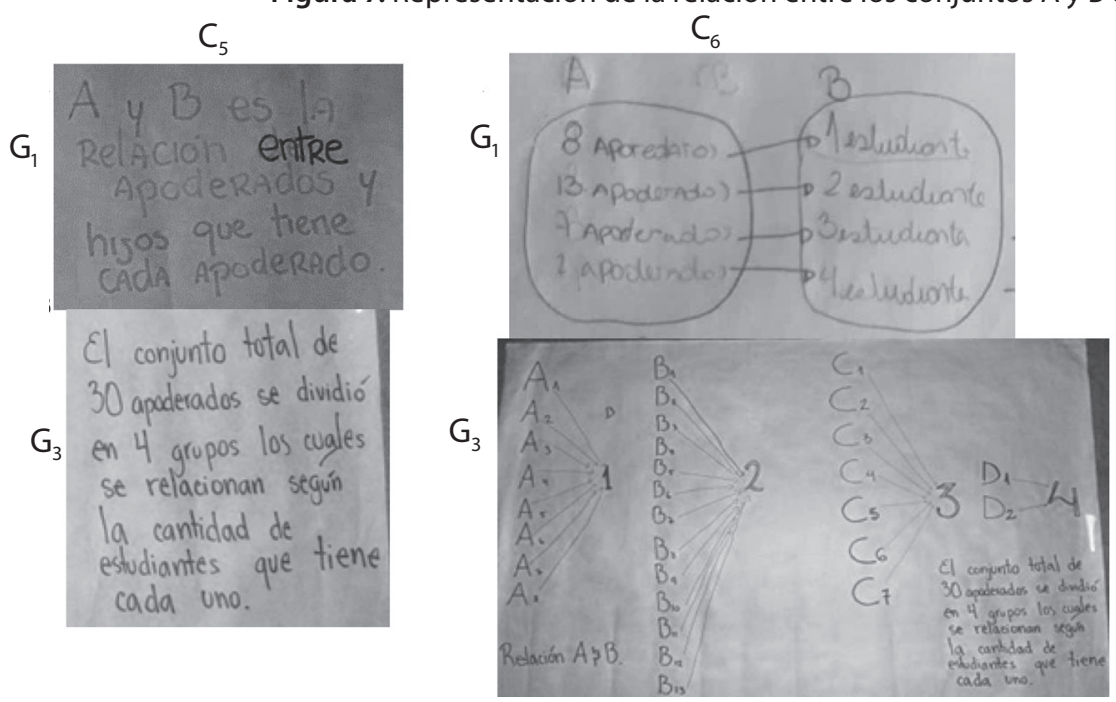

$\mathrm{C}_{7}$

Fuente: Elaboración propia. 
También tres grupos (59\%), $G_{1}, G_{2}$ y $G_{4}$, identificaron la distribución de probabilidad asociada a la variable aleatoria, dos de estos $\left(G_{1}\right.$ y $\left.G_{4}\right)$ representaron la relación entre los conjuntos $B$ y $C$ en lenguaje verbal $\left(C_{8}\right)$, además $G_{1}$ representó la relación en lenguaje figural a través de un diagrama sagital $\left(C_{9}\right)$ como se aprecia en la Figura 8. La respuesta de $G_{2}$ (Figura 8 , derecha) ha sido clasificada en la categoría $C_{10}$, debido a que se identifican elementos de la estrategia dos, por ejemplo, una de las columnas de la tabla corresponde a la probabilidad, aunque en ella fue representada la función compuesta entre el espacio muestral y la probabilidad. Mientras que $G_{5}$ no logró establecer la relación entre los conjuntos $B$ y $C$. Los resultados logrados sobre determinar la distribución de probabilidad de una variable aleatoria son similares a los obtenidos por Flores et al. (2014).

Figura 8. Representación de la relación entre los conjuntos B y C en los grupos

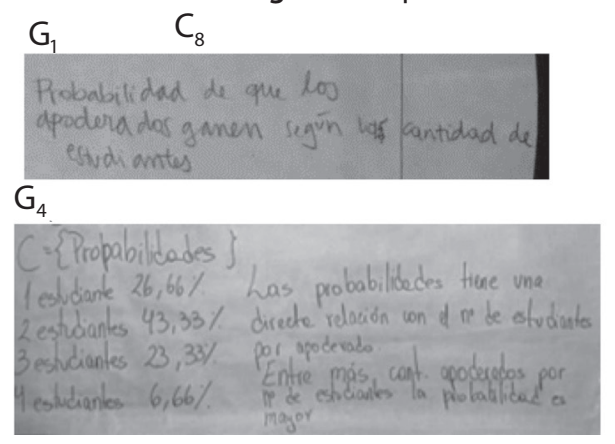

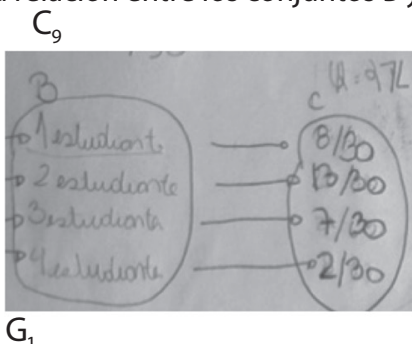

Por ejemplo la probabilidad de un apoderado que tiene un hijo gane la rifa".

Fuente: Elaboración propia.

A partir de las evidencias, en esta fase de la situación a-didáctica, nuevamente se puede constatar el papel que jugó el input (dado de manera consciente y fundamentada en la TRRS), entregado por el docente en la fase de acción para que sus estudiantes hicieran uso de diversas representaciones (TRSS), a fin de que pudiesen formular sus respuestas frente al problema. En ese sentido, se concuerda con Prada-Núñez et al. (2017) sobre la importancia del uso de distintas representaciones para el concepto de función que está involucrado en la situación. También parece ser que, en esta etapa, influyó la devolución realizada por el docente para avanzar en la situación de acción, jugando un papel importante el conocimiento que tuvo el docente sobre el tipo de devoluciones (y la teoría TSD que está detrás de ella) que debe hacer en el aula, para el logro de los objetivos.

\section{Situación validación}

En la presente fase, primero el profesor reconoció que un grupo $\left(\mathrm{G}_{5}\right)$, no logró dar solución al problema, tres grupos propusieron una respuesta parcialmente correcta $\left(G_{2}, G_{3}\right.$ y $\left.G_{4}\right)$ y uno $\left(G_{1}\right)$ respondió completamente bien. Luego, por motivo de duración de la clase, él eligió algunos grupos para exponer y debatir con el curso, la estrategia que utilizaron en la resolución del problema: primer y segundo grupo $\left(G_{3}\right.$ y $\left.G_{2}\right)$ con respuestas incompletas, y un tercero $\left(G_{1}\right)$, con respuesta completa correcta. El profesor moderó la discusión del grupo curso, planteando interrogantes a cada uno, con el propósito de evidenciar las estrategias usadas por estos, sus diferencias y similitudes, por ejemplo: ¿Cómo determinaron la(s) relación(es)? ¿Cómo encontraron los elementos del conjunto $C$ ? ¿Es posible representar en un solo esquema/dibujo/tabla las dos relaciones? ¿Existe alguna diferencia o similitud entre esta estrategia y la del grupo $\mathrm{G}_{1} / \mathrm{G}_{2} / \mathrm{G}_{3}$ ?

Particularmente sobre la interrogante, ¿cómo determinaron la relación entre A y B?, uno de los integrantes del grupo $G_{2}$ respondió considerando las representaciones figurales que habían construido, como se ilustra en la Figura 9. Eso da evidencia del papel que juegan las representaciones para justificar y 
construir las relaciones entre los conjuntos $y$, a la postre, los conceptos de variable aleatoria y distribución de probabilidad que están detrás de ellas. Esto ratifica las afirmaciones provenientes desde la teoría de TRRS en relación con el uso de estas en la enseñanza de la matemática.

Figura 9. Respuesta de $\mathrm{G}_{2}$ ante la pregunta ¿cómo determinaron la relación entre $A$ y $B$ ?

El conjunto de apoderados son 30, y lo dividimos en cuatro grupos según la cantidad de alumnos que tienen en el taller. Como decía en la tabla, el primer grupo con ocho apoderados que los representamos como $A_{1}, A_{2}$ hasta $A_{8}$, solamente tienen un alumno en el taller; en el segundo grupo representamos los otros 13 apoderados que tienen dos alumnos. Luego en el tercer grupo, siete apoderados que tienen tres alumnos y finalmente, el cuarto grupo dos apoderados que tienen cuatro alumnos en el taller.

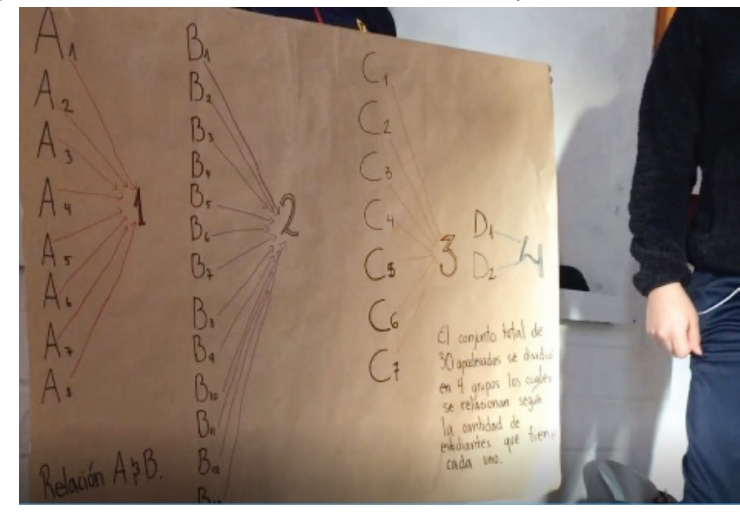

Fuente: Elaboración propia.

En relación con la pregunta ¿cómo determinaron la relación entre $B$ y $C$ ? $G_{1}$ señaló lo expuesto en la Figura 10.

Figura 10. Respuesta de $\mathrm{G}_{1}$ ante la pregunta ¿cómo determinaron la relación entre $\mathrm{B}$ y C?

Determinamos la probabilidad de que un apoderado gane, según la cantidad de alumnos que tiene inscrito en el taller, por ejemplo, un apoderado que tiene un alumno, tiene probabilidad $\frac{8}{30}$ de ganar.

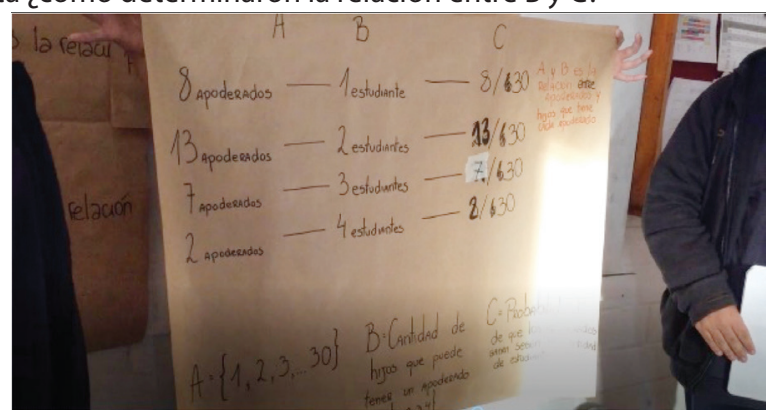

Fuente: Elaboración propia.

Luego, respecto a la interrogante ¿existe alguna diferencia o similitud entre la estrategia del grupo $G_{1}$ y el grupo $G_{2}$ ? Al respecto, un integrante del grupo $G_{4}$ es capaz de observar las diferencias y similitudes como se ilustra en la Figura 11. De esta manera, el grupo curso logró un consenso, afirmando que la respuesta correcta al problema fue la elaborada por $\mathrm{G}_{1}$, cuya respuesta se basó en la estrategia $3\left(\mathrm{C}_{6}\right.$ y $\left.\mathrm{C}_{9}\right)$. Este momento de la clase permite evidenciar la relevancia que tiene en la fase de validación, la gestión del docente (a partir de su conocimiento de la TSD), para ayudar al estudiantado a clarificar ideas y llegar a consensos.

Figura 11. $G_{4}$ comparte con el grupo curso su respuesta a pregunta planteada por el profesor

La similitud es que en las dos está representada la relación entre los conjuntos $A$ y $B$, solo que está escrita de distinta forma. La diferencia es que solo el grupo uno determinó la relación entre $B$ y $C$, y pusieron las probabilidades del conjunto $C$.

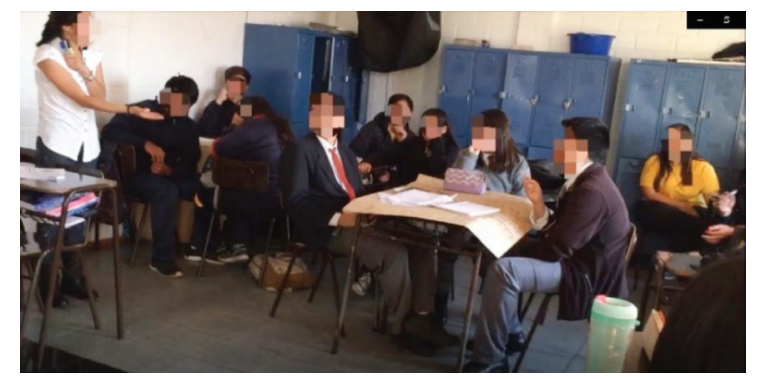

Fuente: Elaboración propia. 
También en este momento de la clase fue posible observar que la estrategia 3 expuesta en las Figuras 9 y 10 "uso de lenguaje figural para representar la variable aleatoria" $\left(C_{6}\right)$, utilizada por los grupos $G_{2}$ y $G_{1}$, respectivamente, facilitó que los estudiantes visualizaran la partición del espacio muestral, conjunto $A$, y la correspondencia de cada uno de sus elementos con el conjunto $B$, contrario a los resultados obtenidos por Fernández, Andrade, Montañez, Beltrán y Zamora (2011), quienes señalan que en este contexto el uso del diagrama sagital genera dificultades.

\section{Institucionalización}

En esta última fase, el profesor empleó la estrategia elaborada por $G_{1}$, centrada en las categorías $C_{6}$, y $C_{9}$ y por medio de las ideas que previamente compartieron los estudiantes, mostró la correspondencia entre los elementos de los conjuntos A y B, además de B y C. Posteriormente, el profesor manifestó al grupo de curso algunas preguntas como: ¿La relación entre los elementos de dos conjuntos del desafío podría ser una función?; ¿Por qué? ¿Qué característica debe tener una relación de conjuntos para que sea una función? Una de los argumentos propuestos por $G_{1}$ y $G_{2}$ fue "la relación entre el conjunto $A$ y $B$ es función, porque a cada elemento del conjunto de partida, conjunto $A$, le corresponde un único elemento del conjunto de llegada, conjunto B, lo mismo ocurre entre los conjuntos B y C". De esta manera, el profesor a partir de las ideas que emergieron en los estudiantes, institucionalizó los conceptos de variable aleatoria y distribución de probabilidad. La Figura 12 presenta algunos momentos de la fase de institucionalización llevada a cabo, donde se resaltó la estrategia $3\left(C_{6}\right.$ y $\left.C_{9}\right)$.

Figura 12. Profesor institucionaliza el saber construido por los estudiantes

La función que relaciona los elementos del conjunto $A$ con $B$, en el contexto de probabilidad, se llama variable aleatoria. Su dominio es el espacio muestral de un experimento aleatorio y su recorrido, un subconjunto de los números reales. Por otra parte, la función que relaciona los elementos del conjunto B con $C$, también en el contexto de probabilidad posee un nombre particular, función de probabilidad o distribución de probabilidad. Su dominio es el recorrido de una variable aleatoria y su recorrido, son las probabilidades asociadas a cada valor de aquella variable.

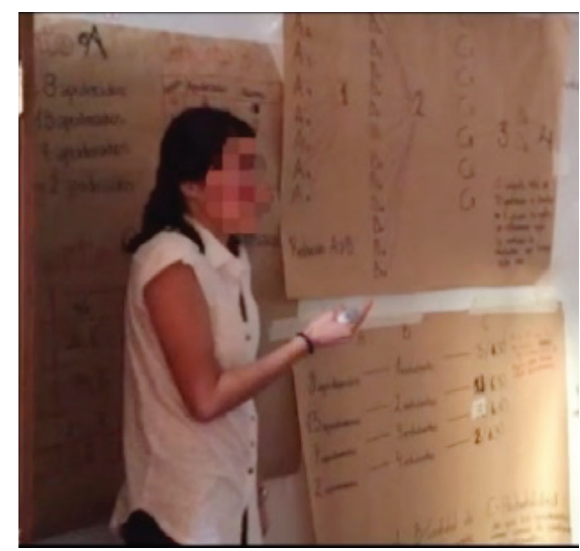

Fuente: Elaboración propia.

Así, el profesor formalizó el saber considerando las ideas de los grupos, planteadas en la fase de validación; por un lado, las representaciones usadas por los estudiantes, y por otro, las diferentes respuestas y consensos logrados por ellos, en la fase de validación. Esto pone de relieve el papel que jugaron las teorías didácticas que estuvieron detrás de la clase, tanto desde el conocimiento del profesor como en el diseño de la propuesta de aula y su estructura. 


\section{SÍNTESIS Y REFLEXIONES FINALES}

El estudio desarrollado permitió valorar un proceso de instrucción, en torno a la variable aleatoria y su distribución de probabilidad en estudiantes de secundaria, fundamentado en la Teoría de Situaciones Didácticas (TSD) y la Teoría de Registro de Representaciones Semiótica (TRRS). En este proceso, se evidenció el papel que jugó para el aprendizaje de sus aprendices, la construcción de la propuesta de instrucción con base en ambas teorías, ya que, por un lado, la TSD les permitió ir construyendo su saber en un entorno colaborativo, donde el docente jugó el papel de mediador del aprendizaje y sus devoluciones permitieron avanzar en el caso de estancamiento. Por otro lado, considerar la teoría TRRS ha permitido reconocer elementos de la variable aleatoria y función de probabilidad con mayor facilidad, dada su naturaleza funcional y el papel que juegan estos en su construcción.

Sobre el primer objeto matemático, más de la mitad de los estudiantes (64\%) logró identificarlo y representarlo en lenguaje verbal, figural o tabular, similar a lo que se vivenció con el segundo objeto señalado (59\%). Las diversas estrategias propuestas por los participantes concuerdan con los distintos registros de representación de los conceptos en cuestión, que han sido observados en investigaciones previas (Doukhan y Gueudet, 2019; Landín y Salinas, 2016). Aquello, en términos de la TRRS, podría indicar que los estudiantes están en vías de construcción de los objetos variable aleatoria y distribución de probabilidad, donde esta presenta su vinculación con el objeto función y que debe poner en juego los estudiantes.

Desde la TSD, también fue posible observar el importante rol que cumplió el profesor en las fases de una situación didáctica. En la situación acción, en un inicio realizó preguntas de devolución previstas en el diseño del proceso de instrucción, debido a que los estudiantes identificaron incorrectamente el espacio muestral; entonces, por medio de interrogantes guió su trabajo y permitió subsanar la dificultad. En la situación de validación, a partir de preguntas, el docente dirigió el debate entre los cinco grupos del curso. En la fase de institucionalización, formalizó el saber construido por los estudiantes. Por tanto, en este estudio se ha constatado que el conocimiento de la matemática y su didáctica por parte del profesor, es fundamental en el proceso de construcción del conocimiento en los estudiantes (Yang, 2014).

Es importante destacar cómo el proceso de instrucción ayudó al profesor (y en consecuencia a sus estudiantes), a identificar dificultades en la comprensión del significado o uso de los objetos estudiados; por ejemplo, cuando entrega oportunamente la pregunta de devolución. Se sostiene que el uso articulado de las dos teorías TSD y TRRS han sido clave en este proceso, por lo que una sugerencia que puede surgir de este estudio para la comunidad académica, va encaminada en la propuesta articuladora de teorías didácticas que favorezcan los procesos de enseñanza y aprendizaje, donde el profesor debe ser consciente de estos elementos teóricos como herramientas poderosas para el logro de sus objetivos de aprendizaje, que vayan más allá de una memorización de fórmulas o técnicas matemáticas (que están detrás de la variable aleatoria y función de probabilidad), y que los lleva a la comprensión de las mismas a partir de un contexto cotidiano.

Finalmente, es posible concluir que este proceso de instrucción parece haber permitido a los diferentes actores que participaron en él, avanzar en el aprendizaje y la enseñanza de la variable aleatoria y función de probabilidad, en el sentido que permitió al profesor, abandonar su rol de comunicador del saber y a los estudiantes construir su propio conocimiento como una experiencia grupal y colaborativa, con la cual el docente pudo conectar el saber institucional. De esta forma, se proyecta como un recurso valioso para profesores, tanto en ejercicio como en formación, que deban enseñar la variable aleatoria y/o distribución de probabilidad, pues la metodología de trabajo que se empleó (diseño de la situación didáctica, implementación y análisis de esta), es recomendada por Sánchez y Gómez-Blancarte (2011) a los docentes para el aprendizaje de nociones de estadística, de forma que ellos son capaces de aplicar tal aprendizaje en el aula. Dentro de las líneas de investigación del estudio, se destacan las necesidades 
de estudiar, el diseño y puesto en juego de otras propuestas de instrucción que articulen diferentes teorías didácticas (quizás bajo la metodología de experimentos de enseñanza), para observar cómo estas influyen en los procesos de enseñanza y aprendizaje. Por otro lado, parece ser interesante indagar cómo docentes que no tienen conocimiento de la TSD y/o de la TRRS, ponen en juego la propuesta de instrucción y qué resultados obtienen en el alumnado.

La TSD y la TRRS han permitido diseñar y analizar una propuesta de aula para dos objetos de enseñanza complejos. Estudiar, desde teorías didácticas, cómo llevar al aula objetos difíciles de enseñar puede ayudar a profesores en ejercicio y en formación, a mirar su práctica con mayor fundamento, a fin de hacerla más accesible y comprensible a sus estudiantes.

\section{REFERENCIAS}

Acosta, M., Monroy, L. y RuedA, K. (2010). Situaciones a-didácticas para la enseñanza de la simetría axial utilizando Cabri como medio. Revista Integración, 28(2), 173-189.

Batanero, C. (2001). Didáctica de la estadística. Granada, España: Universidad de Granada.

Batanero, C. (2004) Ideas estocásticas fundamentales ¿qué contenido se debe enseñar en la clase de probabilidades? En J. Fernández, M. Sousa y S. Ribeiro (Eds.), Actas do I Encontro de Probabilidades e Estatística na Escola (pp. 9-30). Braga, Portugal: Centro de Investigação em Educação da Universidade do Minho.

BIzET, V. (2017). Una secuencia didáctica para el aprendizaje del concepto de variable aleatoria desde la teoría de situaciones didácticas (Tesis de maestría). Pontificia Universidad Católica de Valparaíso, Chile.

Brousseau, G. (1998). Théorie des Situations Didactiques. Grenoble, Francia: La pensée sauvage.

Brousseau, G. (2007). Iniciación al estudio de la teoría de las situaciones didácticas. Buenos Aires, Argentina: Libros del Zorzal.

DoukHAN, C. y GueUdet, G. (2019). Students' difficulties at the secondary-tertiary transition: the case of random variables. En U. Jankvist, M. van den Heuvel-Panhuizen y M. Veldhuis (Eds.), Proceedings of the Eleventh Congress of the European Society for Research in Mathematics Education (pp. 24642471). Utrecht, Países Bajos: Freudenthal Group y Freudenthal Institute of the Utrecht University y ERME.

Duval, R. (2006). Un tema crucial en la educación matemática: la habilidad para cambiar el registro de representación. Gaceta de la Real Sociedad Matemática Española, 9(1), 143-168.

Fernández, F.; Andrade, L.; Montañez, J.; Beltrán, J. y Zamora, S. (2011). Hacia una posible aproximación comprensiva de la variable aleatoria. Trabajo presentado en la XIII Conferência Interamericana de Educação Matemática. Recife, Brasil: CIAEM.

FLıCK, U. (2004). Introducción a la investigación cualitativa. Madrid, España: Ediciones Morata.

Flores, B.; García, J. y SÁnChez, E. (2014). Avances en la calidad de las respuestas a preguntas de probabilidad después de una actividad de aprendizaje con tecnología. En M. González, M. Codes, D. Arnau y T. Ortega (Eds.). Investigación en Educación Matemática XVIII (pp. 307-316). Salamanca, España: SEIEM.

Heitele, D. (1975). An epistemological view of fundamental stochastic ideas. Educational Studies in Mathematics, 6, 187-205.

Hernández, R.; Fernández, C. y Baptista, P. (2006). Metodología de la investigación. Ciudad de México, México: McGraw-Hill. 
LANDIN, P. y SALINAS, J. (2016). Probabilistic reasoning of high school students on sample space and probability of compound events. Trabajo presentado en el 13th International Congress on Mathematical Education. Hamburgo, Alemania: ICMI.

National Council of Teachers of Mathematics (2000). Principles and standards for school mathematics. Reston, Estados Unidos: NCTM.

Ministerio de Educación de Chile (2015). Bases Curriculares $7^{\circ}$ básico a $2^{\circ}$ medio. Gobierno de Chile. Santiago, Chile: Unidad de Currículum y Evaluación.

Ministerio de Educación de Chile (2016). Matemática. Programa de estudio segundo medio. Gobierno de Chile. Santiago, Chile: Unidad de Currículum y Evaluación.

Ministerio de Educación, Cultura y Deporte (2015). Real Decreto 1105/2014, de 26 de diciembre, por el que se establece el currículo básico de la Educación Secundaria Obligatoria y del Bachillerato. Boletín Oficial del Estado, 3, 135.

Prada-Núnez, R.; Hernández-Suárez, C. y Jaimes, L. (2017). Representación semiótica de la noción de función: concepciones de los estudiantes que transitan del colegio a la universidad. Panorama, 11(20), 34-44.

SALAZAR, R. (2014). La variable aleatoria con probabilidad desde la perspectiva de la teoría APOE (Tesis de maestría). Pontificia Universidad Católica de Valparaíso, Chile.

Sánchez, E. y Gómez-Blancarte, A. L. (2011). El desarrollo del pensamiento estadístico de profesores de secundaria en servicio. En J. Godino (Ed.), Investigaciones actuales en Educación Estadística y Formación de Profesores (pp. 55-72). Granada, España: Universidad de Granada.

YANG, X. (2014). Conception and characteristics of expert mathematics teachers in China. Wiesbaden, Alemania: Springer. 